\title{
Degradation of HK2 by chaperone-mediated autophagy promotes metabolic catastrophe and cell death
}

\section{Citation}

Xia, H., A. Najafov, J. Geng, L. Galan-Acosta, X. Han, Y. Guo, B. Shan, et al. 2015. “Degradation of HK2 by chaperone-mediated autophagy promotes metabolic catastrophe and cell death." The Journal of Cell Biology 210 (5): 705-716. doi:10.1083/jcb.201503044. http://dx.doi.org/10.1083/ jcb.201503044.

\section{Published Version}

doi:10.1083/jcb.201503044

\section{Permanent link}

http://nrs.harvard.edu/urn-3:HUL.InstRepos:25658325

\section{Terms of Use}

This article was downloaded from Harvard University's DASH repository, and is made available under the terms and conditions applicable to Other Posted Material, as set forth at http:// nrs.harvard.edu/urn-3:HUL.InstRepos:dash.current.terms-of-use\#LAA

\section{Share Your Story}

The Harvard community has made this article openly available.

Please share how this access benefits you. Submit a story.

Accessibility 


\title{
Degradation of HK2 by chaperone-mediated autophagy promotes metabolic catastrophe and cell death
}

\author{
Hong-guang Xia, ${ }^{1}$ Ayaz Najafov, ${ }^{1 *}$ Jiefei Geng, ${ }^{1 *}$ Lorena Galan-Acosta, ${ }^{2 *}$ Xuemei Han, ${ }^{3 *}$ Yuan Guo, ${ }^{4}$ \\ Bing Shan, ${ }^{4}$ Yaoyang Zhang, ${ }^{4}$ Erik Norberg, ${ }^{2}$ Tao Zhang, ${ }^{4}$ Lifeng Pan, ${ }^{5}$ Junli Liu, ${ }^{5}$ Jonathan L. Coloff, ${ }^{1}$ \\ Dimitry Ofengeim, ${ }^{1}$ Hong Zhu, ${ }^{1}$ Kejia Wu, ${ }^{4}$ Yu Cai, ${ }^{4}$ John R. Yates, ${ }^{3}$ Zhengjiang Zhu, ${ }^{4}$ Junying Yuan, ${ }^{1,4}$ \\ and Helin Vakifahmetoglu-Norberg ${ }^{1,2}$

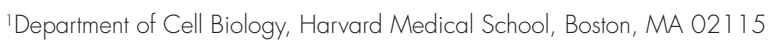

Hexokinase II (HK2), a key enzyme involved in glucose metabolism, is regulated by growth factor signaling and is required for initiation and maintenance of tumors. Here we show that metabolic stress triggered by perturbation of receptor tyrosine kinase FLT3 in non-acute myeloid leukemia cells sensitizes cancer cells to autophagy inhibition and leads to excessive activation of chaperone-mediated autophagy (CMA). Our data demonstrate that FLT3 is an important sensor of cellular nutritional state and elucidate the role and molecular mechanism of CMA in metabolic regulation and mediating cancer cell death. Importantly, our proteome analysis revealed that HK2 is a CMA substrate and that its degradation by CMA is regulated by glucose availability. We reveal a new mechanism by which excessive activation of CMA may be exploited pharmacologically to eliminate cancer cells by inhibiting both FLT3 and autophagy. Our study delineates a novel pharmacological strategy to promote the degradation of HK2 in cancer cells.

\section{Introduction}

Autophagy is an important degradative mechanism that delivers select cytoplasmic components into the lysosome for recycling purposes (Mizushima and Komatsu, 2011). Activation of autophagy promotes cell survival under adverse conditions, such as during metabolic stress. Accordingly, inhibition of autophagy in cancer cells has been proposed as a potential therapeutic strategy (White, 2012). However, because inhibition of autophagy itself is not sufficient to induce cancer cell death, the mechanism and means by which to sensitize cancer cells to autophagy inhibition remain to be identified.

Chaperone-mediated autophagy (CMA) delivers select proteins with a pentapeptide CMA-targeting motif into the lysosome mediated by their binding with the chaperone Hsc70 and the interaction with lysosome-associated membrane protein type $2 \mathrm{~A}$

*A. Najafov, J. Geng, L. Galan-Acosta, and X. Han contributed equally to this paper.

Correspondence to Junying Yuan: jyuan@hms.harvard.edu

Abbreviations used in this paper: 2DG, 2-deoxy-D-glucose; 7N-1, 7-Cl-O-Nec-1 $\mathrm{AML}$, acute myeloid leukemia; $\mathrm{ClQ}$, chloroquine; $\mathrm{CMA}$, chaperone-mediated autophagy; ECAR, extracellular acidification rate; LC-MS, liquid chromatography-mass spectrometry; OCR, oxygen consumption rate; Pl, propidium iodide; RTK, receptor tyrosine kinase; STS, staurosporine; TMT, tandem mass tag; WB, Western blot; wt, wild type.
(Lamp-2A), a lysosomal membrane receptor (Kaushik and $\mathrm{Cu}-$ ervo, 2012). We have shown that autophagy inhibition by a small molecule inhibitor, spautin-1, can lead to the activation of CMA to mediate the degradation of mutant p53 under nutrient deprivation conditions (Vakifahmetoglu-Norberg et al., 2013). Furthermore, we showed that CMA activation induces the death of nonproliferating quiescent cancer cells, while normal cells are spared (Vakifahmetoglu-Norberg et al., 2013). These findings raise the intriguing possibility of inducing CMA activation as a potential anticancer therapy. However, because maximal CMA activation requires a combination of nutritional stress and a blockade of autophagy, the therapeutic feasibility of this strategy is unclear.

Receptor tyrosine kinases (RTKs) are critical mediators of cell growth and survival. Abnormal activation of the PI3K-Akt pathway is common in a wide range of cancers with mutated and dysregulated RTKs. Fms-like tyrosine kinase 3 (FLT3), a member of the class-III RTK family, is a validated target for the treatment of acute myeloid leukemia (AML; Kayser and Levis,

(C) 2015 Xia et al. This article is distributed under the terms of an Attribution-NoncommercialShare Alike-No Mirror Sites license for the first six months after the publication date (see http://www.rupress.org/terms). After six months it is available under a Creative Commons License (Attribution-Noncommercial-Share Alike 3.0 Unported license, as described at http://creativecommons.org/licenses/by-nc-sa/3.0//. 
2014). Quizartinib (AC220), a selective and potent inhibitor of FLT3, is currently under clinical trial against AML with activating FLT3 mutations (Zarrinkar et al., 2009). However, the value of targeting FLT3 beyond AML has not been well explored.

Hexokinase II (HK2) is a key enzyme involved in catalyzing the first committed step of glucose metabolism, regulated by growth factor signaling (Shaw and Cantley, 2006; Nederlof et al., 2014). It has been recognized as an oncogenic kinase, as it is required for tumor initiation and maintenance of multiple types of tumors (Patra et al., 2013). Consequently, inhibition of HK2 has been proposed as a potential anticancer strategy. However, no method has yet been described to reduce HK2 levels in cancer cells.

In this study, we investigated the mechanism that sensitizes cancer cells to autophagy inhibition. We show that inhibition of FLT3 in nonhematopoietic cancers increases their sensitivity to autophagy inhibition under conditions where they are normally resistant. Simultaneous inhibition of FLT3 and autophagy leads to excessive activation of CMA and cancer cell death under normal nutritional conditions. We characterized targets of the CMA pathway using an unbiased proteomic approach and identified HK2, a key glycolytic enzyme, as a CMA substrate. Importantly, we provide a new mechanism by which excessive activation of CMA may be exploited as a method to eliminate cancer cells by inducing metabolic catastrophe and delineate a novel strategy to promote the degradation of HK2 in cancer cells.

\section{Results and discussion}

\section{FLT3 inhibitor ACE20 (Quizartinib) sensitizes nonconfluent cancer cells to spautins under normal nutritional conditions}

Our medicinal chemistry campaign to improve the original autophagy inhibitor spautin-1 (C43) synthesized and tested the biological activities of $>700$ derivatives (unpublished data) and led to the identification of A70, which inhibits autophagy with an $\mathrm{IC}_{50}$ of $0.076 \mu \mathrm{M}$ (Fig. S1 A). Similar to the actions of C43, the treatment with $\mathrm{A} 70$ induces the death of cancer cells under glucose-free as well as confluent conditions (Fig. S1 B); importantly, A70 was able to induce cancer cell death at significantly lower concentrations than that of C43 (Fig. S1 C).

Because both C43 and A70 induce cell death only in glucose-free or confluent conditions, we sought to identify small molecules that could mimic these stressful cellular states and thus, in combination with spautins, would eliminate cancer cells regardless of growth condition. We established a high-throughput screen using ovarian carcinoma-derived ES2 cells, which display resistance to $\mathrm{C} 43$ or A70 under normal proliferating and full nutritional conditions (Fig. S1 B; Vakifahmetoglu-Norberg et al., 2013). We screened 8,248 compounds from the ICCB Known Bioactives Library, and identified 115 hits with potential minimal toxicity when treated alone but that significantly promoted the loss of cell viability in the presence of A70 in both ES2 and Sum159 cells (unpublished data).

This analysis identified Quizartinib (AC220), an RTK inhibitor, as the most effective sensitizer of the autophagy inhibitors A70 or C43. AC220 significantly increased the efficacy of A70 and C43 in inducing cell death of ES2 and Sum159 cells in a dose-dependent manner under normal growth conditions (Fig. 1, A and B), whereas single compound treatment had no or little effect on the cell survival. Similar results were observed in Bcap-37, MCF-7, HCT116, and MDA-MB-435 cancer cells (Fig. $1 \mathrm{C}$ and S1 D). In contrast to the cancer cell lines analyzed, a normal cell line derived from the MDCK was not significantly affected by the combination treatment of C43 and AC220 (Fig. 1 C). In addition, we tested the sensitivity for single or combination treatments of $\mathrm{C} 43$ and AC220 on three AML cell lines (Fig. 1 D). While only Molm-14, an AML cell line carrying FLT3/ITD mutation, died with AC220 alone, the combination treatment of $\mathrm{AC} 220$ and $\mathrm{C} 43$ significantly reduced the cell viability of HEL and OCI-AML3 AML cells, which have no FLT3 mutation (Fig. 1 D). Western blot (WB) analysis of the level of FLT3 showed that the total and phospho-FLT3 levels are expressed in all cell lines used in this study (Fig. S1 E).

Furthermore, while other RTK inhibitors, such as Lapatinib and Nilotinib, could also promote the death of ES2 cells in the presence of $\mathrm{C} 43$, these compounds induced substantial death even when treated alone (Fig. S1 F), which suggests that AC220 provides the chemical means to selectively sensitize cancer cells to autophagy inhibition under normal growth conditions. Because the susceptibility of both $\mathrm{C} 43$ and A70 is sensitized by the effect of AC220, we used both compounds in subsequent experiments.

Consistent with the inhibition of FLT3, silencing the FLT3 expression mimicked the effect of AC220: knockdown of FLT3 had no effect on the survival of ES2 cells, whereas it markedly increased the cell death sensitivity to spautins and significantly reduced cell viability in combination with either $\mathrm{C} 43$ or A70 (Fig. 1 E). Thus, inhibition of FLT3 by AC220 or its knockdown is sufficient to enhance the effect of spautins to induce the death of cancer cells. Notably, we detected reduced levels of FLT3 autophosphorylation in ES2 cells under confluence and in glucose-free conditions compared with that of normal culture condition (Fig. 1 F), which could potentiate the effect of spautins. Accordingly, the application of AC220 in normal proliferating culture conditions mimicked FLT3 inhibition observed under metabolic stress conditions, suggesting that the FLT3 signaling pathway is a critical sensor and regulator of cellular nutritional states. To further assess the contribution of autophagy inhibition in combination of AC220, we performed siRNA-mediated knockdown of $A T G 7$, an essential autophagy-related gene, in ES2 cells, which led to a marked decreased in viability of ES2 cells in combination with AC220 (Fig. $1 \mathrm{G}$ ).

C43 treatment of ES2 cells in confluence leads to the degradation of multiple deubiquitinating enzymes through the proteasomal pathway (Liu et al., 2011; Vakifahmetoglu-Norberg et al., 2013). To test if AC220 treatment mimics cellular confluence, we treated nonconfluent cells ES2 and HCT116 with AC220 and C43 and found that the levels of endogenous Beclin1, USP10, and USP13 also progressively decreased in a concentration-dependent manner. The reduction of the levels of these proteins was strongly correlated with the inhibition of FLT3 autophosphorylation by AC220 (Figs. $1 \mathrm{H}$ and S1 G). In contrast, the treatment of C43 or AC220 alone had no effect on USP10, USP13, or Beclin1 protein levels under nonconfluent conditions (Fig. $1 \mathrm{H}$ ).

\section{FLT3 inhibition suppresses glycolysis and induces macroautophagy}

FLT3 has a well-established role in regulating the survival and proliferation of hematopoietic stem/progenitor cells. Activation of FLT3 kinase leads to signal transduction of several downstream pathways, such as the PI3K-AKT pathway and the RAS/ 
A

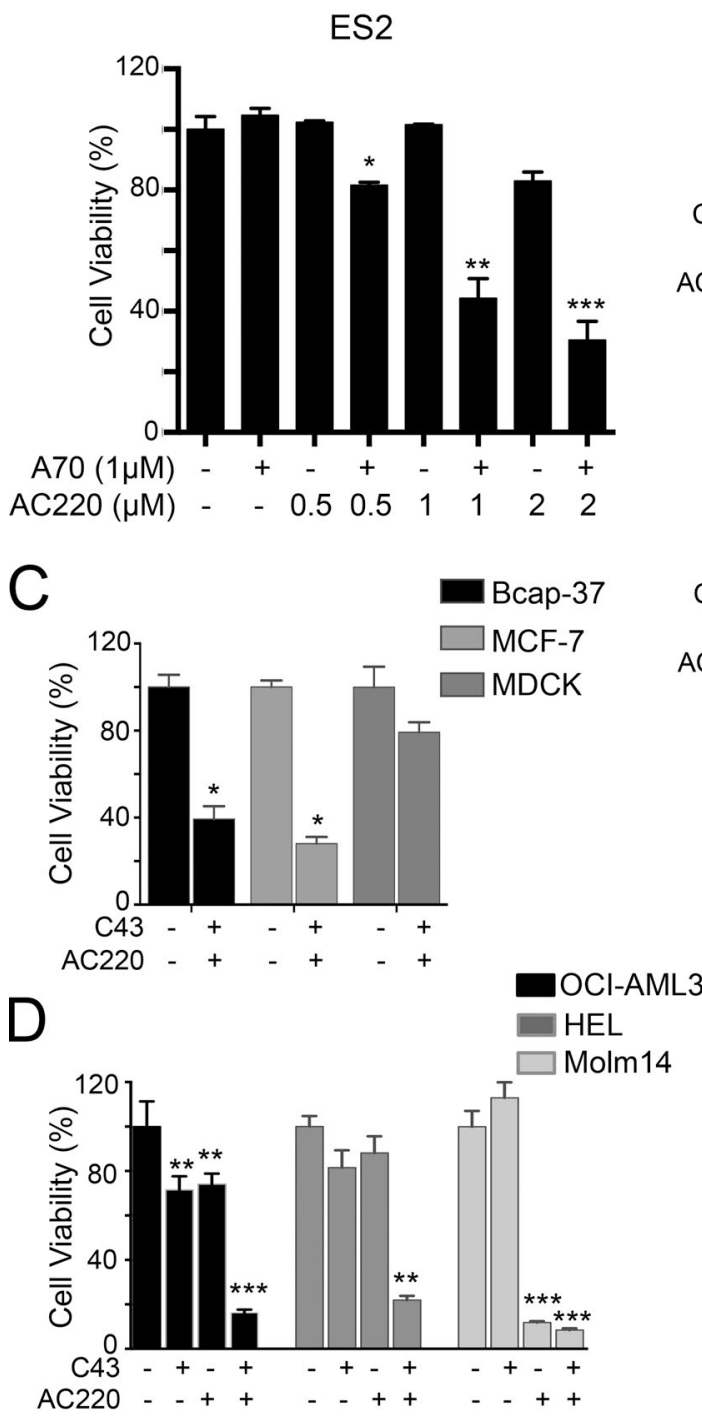

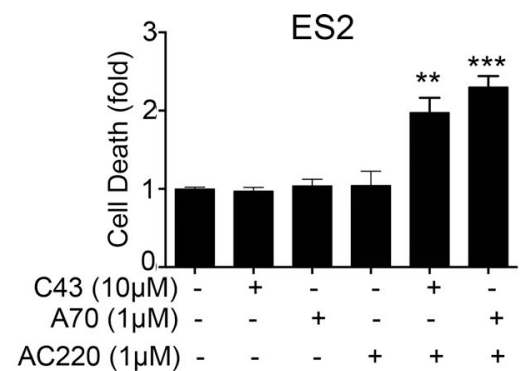

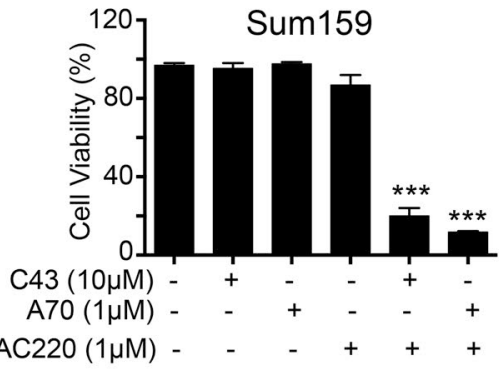

$\mathrm{E}$
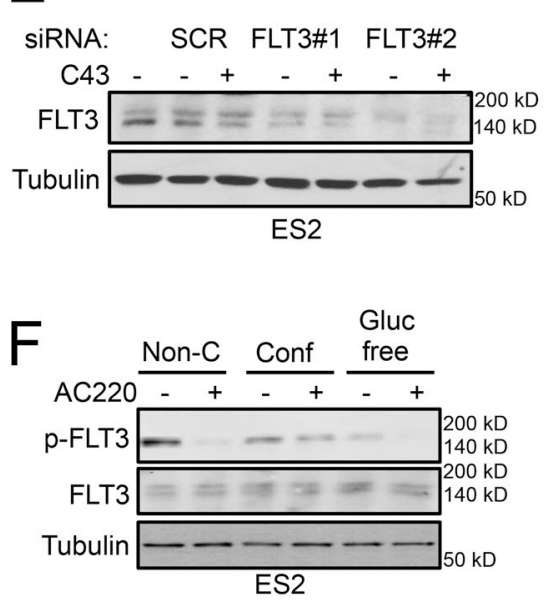

$\mathrm{AC} 220(1 \mu \mathrm{M})$
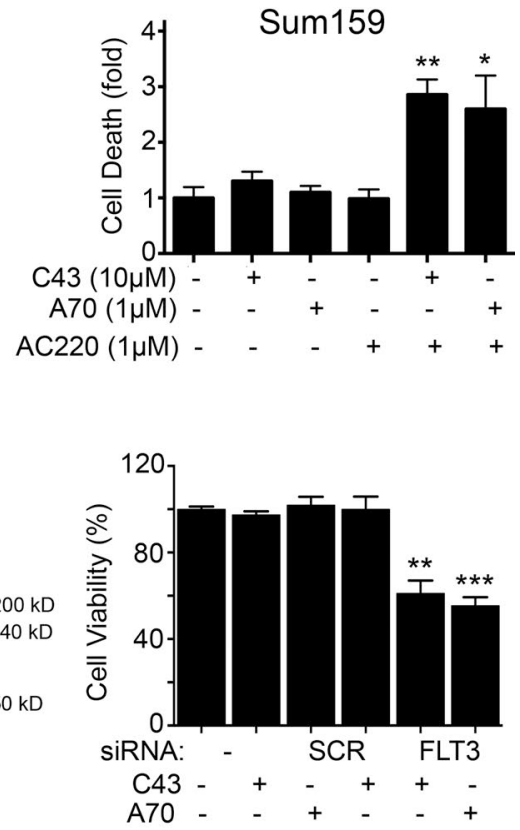

\section{G}

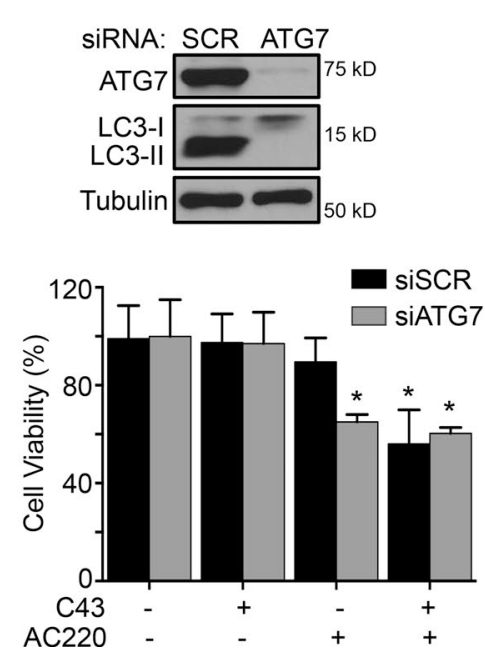

$\mathrm{H}$
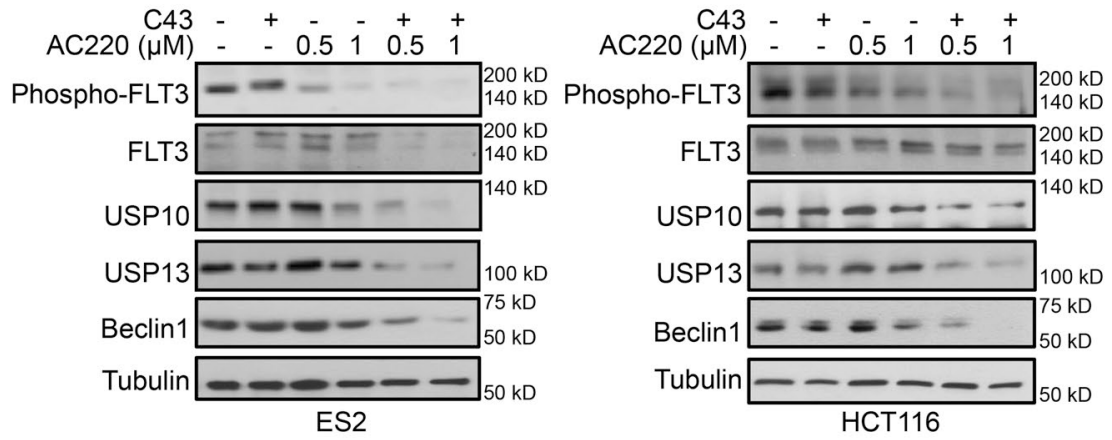

Figure 1. FLT3 inhibition sensitized nonconfluent cancer cells to spautins. (A) Cell viability (\%) of ES2 cells treated with increasing concentrations of $A C 220$ and/or A70 for $24 \mathrm{~h}$. (B) Cell viability (\%) and cell death (fold) of ES2 and Sum 159 cells treated with AC220 and/or C43 or A70 for 24 h. (C and D) Cell viability (\%) of Bcap-37, MCF-7, and MDCK cells (C) and of OCI-AML3, HEL, and Molm-14 cells (D), treated with AC220 and C43 for 24 h. (E) Cell viability (\%) of scramble (SCR) or FLT3 siRNA-transfected ES2 cells treated with C43 for 24 h. WB confirmed the FLT3 siRNA efficiency. (F) Phospho- and 
RAF/MEK/ERK cascade (Parcells et al., 2006). To uncover the mechanism by which AC220 conveys sensitivity toward inhibition of autophagy, we explored the downstream consequences of inhibiting the FLT3 pathway in nonhematopoietic lineagederived cells, such as breast and ovarian carcinoma cells. Toward this end, we assessed cellular proliferation and found that AC220 can suppress the proliferation of ES2 and Sum159 cells without inducing cell death (Fig. 2 A). AC220-treated cells also demonstrated a time-dependent reduction of Akt phosphorylation on Ser473, a marker for its activation (Correa et al., 2012; Fig. 2 A). Treatment with C43 alone induced a slight increase in Akt Ser473 phosphorylation, which was inhibited by AC220 (Fig. 2 B, left). Notably, Akt Ser473 phosphorylation was inhibited in ES2 cells cultured under confluent conditions (Fig. $2 \mathrm{~B}$, right), suggesting that inhibition of Akt activation by AC220 might contribute in part to its sensitization to spautins in nonconfluent cell cultures.

Metabolic reprogramming as a result of oncogenic signaling alteration plays an important role in regulating energy and biomaterial production in cancer cells. However, the role of FLT3 in glucose metabolism is not well appreciated. Given that Akt has a crucial role in regulating glucose metabolism (Elstrom et al., 2004), we measured lactate production in nonconfluent cells in the presence of $\mathrm{AC} 220$ or in combination with C43. In both ES2 and Sum159 cells, AC220 was able to markedly reduce lactate production and significantly block glucose uptake, which was further reduced in the presence of C43 (Fig. $2 \mathrm{C}$ and Fig. S2 A). Our investigation of the effect of AC220 and C43 on the extracellular acidification rate (ECAR), which measures the rate of lactate production from glycolysis, showed that while $\mathrm{C} 43 \mathrm{did}$ not affect glycolysis, both glycolytic function and the glycolytic capacity were significantly suppressed by AC220 treatment and further reduced in combination with C43 (Fig. 2 D). Consistently, ECAR analysis on Molm-14 and OCI-AML3 cells revealed that the glycolysis and glycolytic capacity were markedly decreased by both single AC220 and combination treatment with $\mathrm{C} 43$ in AML cell lines similar to that of ES2 ovarian cancer cells (Figs. $2 \mathrm{E}$ and S2 B). We next characterized the impact of AC220 and C43 on the cellular glycolytic state in ES2 cells by glucose flux analysis. This analysis revealed that all quantitatively validated individual intracellular metabolic products of glucose from glycolysis were significantly suppressed by AC220 treatment and further reduced in combination with C43 (Fig. 2 F).

Collectively, our data from ECAR, glucose uptake, and flux analyses demonstrate that AC220 significantly inhibits glycolysis, suggesting that FLT3 may have a previously unappreciated role in regulating glucose uptake and glycolysis in cancer cells beyond AML, and that the FLT3 inhibitor AC220 may be considered for the treatment of non-AML related cancers in combination therapies.

Consistent with blockage of glucose metabolism, treatment of AC220 increased the levels of LC3-II, which is indicative of autophagy activation, in ES2 and Sum159 cancer cells (Figs. $2 \mathrm{G}$ and S2 C). As expected, C43 or A70 were able to attenuate the autophagy up-regulation induced by AC220
(Fig. 2 G). In contrast, although Lapatinib or Nilotinib were quite effective in inducing ES2 cell death (Fig. S1 F), their effect on glucose uptake was significantly less compared with that of AC220 (Fig. S2 D). Furthermore, increased LC3-II levels were only detected in Lapatinib-treated cells, whereas no change was found upon Nilotinib treatment. Autophosphorylation of FLT3 was not reduced by either Lapatinib or Nilotinib (Fig. S2 D). Consistent with the importance of FLT3 in mediating cell death in this context, dual suppression of Akt and PI3K, the downstream mediators of FLT3, elicited robust cell death when combined with C43 treatment (Fig. S2 E). Additionally, a significant autophagy induction was not detected in the presence of PI3K inhibitors (LY294002 or Wortmannin) or Akt inhibitor MK2206 alone (Fig. S2 F). Collectively, these findings indicate that FLT3 has a role as an important sensor of cellular nutritional state in cancer cells beyond AML and that its inhibition promotes metabolic stress and strong dependency on autophagy for survival.

\section{Combinatory inhibition of FLT3 and autophagy activates CMA}

C43 treatment under nutritional limiting or confluent conditions significantly accelerates the activation of CMA, which leads to the degradation of mutant p53 (Vakifahmetoglu-Norberg et al., 2013). We found that the combination of AC220 with either C43 or A70 treatment can similarly enhance the degradation of mutant p53 proteins in nonconfluent conditions in ES2 cells (Figs. $3 \mathrm{~A}$ and S3 A), whereas no significant change of p53 mRNA levels were observed (Fig. S3 B). The level of degradation of mutant p53 upon $\mathrm{AC} 220$ and $\mathrm{C} 43$ was comparable with the effect of C43 treatment observed in confluent conditions (Fig. S3 C). Significant reduction of mutant p53 in MDA-MB-231, Sum159, and MDA-MB-435 cell lines was also observed upon combination treatment (Fig. $3 \mathrm{~A}$, bottom). In contrast, single treatment of $\mathrm{C} 43, \mathrm{~A} 70$, or $\mathrm{AC} 220$ had no effect on the mutant p53 protein levels under nonconfluent conditions (Figs. $3 \mathrm{~A}$ and S3, A and C). The knockdown of FLT3 levels did not affect the levels of mutant $\mathrm{p} 53$ protein, but subsequent treatment with C43 induced degradation of mutant p53 (Fig. 3 B). Furthermore, while the proteasome inhibitor MG132 did not show an effect on the levels of mutant p53 protein, lysosomal inhibitor chloroquine (ClQ) attenuated AC220 and C43 combination-induced mutant p53 degradation in ES2 cells (Fig. 3 C), suggesting that mutant p53 is degraded through the lysosomal pathway. The reduction in mutant p53 levels upon AC220 and C43 treatment was accompanied by increased degradation of GAPDH and IкB, two known substrates of CMA (Aniento et al., 1993; Cuervo et al., 1998; Fig. 3 D). Additionally, siRNA-mediated knockdown of either Hsc70 or Lamp2A, the key mediators of CMA, in ES2 cells attenuated mutant p53 depletion induced by the combination treatment (Fig. $3 \mathrm{E}$ ), demonstrating that inhibition of FLT3 by AC220 in combination with C43 activates CMA in proliferating cells under nutritionally rich conditions.

Next, we explored the mechanism of cell death induced by the combination treatment of AC220 and C43. While single compound treatment had only a minimal effect, the combi-

total FLT3 protein levels of ES2 cells cultured in confluent (Conf), nonconfluent (Non-C), or glucose-free (Gluc free) conditions, treated with AC220 for $24 \mathrm{~h}$. (G) Cell viability (\%) of scramble (SCR) or ATG7 siRNA-transfected ES2 cells treated with AC220 or C43 for $24 \mathrm{~h}$. (H) WB of indicated proteins in ES2 and $\mathrm{HCT} 116$ cells treated with increasing concentrations of AC220 and/or C43 for $24 \mathrm{~h}$. Anti- $\alpha$-tubulin was used as a loading control. Cells were treated with $0.1 \%$ DMSO (control: vehicle) or $1 \mu \mathrm{M} \mathrm{AC220}$ and $10 \mu \mathrm{M} \mathrm{C43}$, unless otherwise stated. In all the experiments, treatment groups were compared with control group, unless otherwise indicated. Error bars indicate $\pm \mathrm{SD}$. ${ }^{*}, \mathrm{P}<0.05 ;{ }^{* *}, \mathrm{P}<0.01 ;{ }^{* * *}, \mathrm{P}<0.001$. 

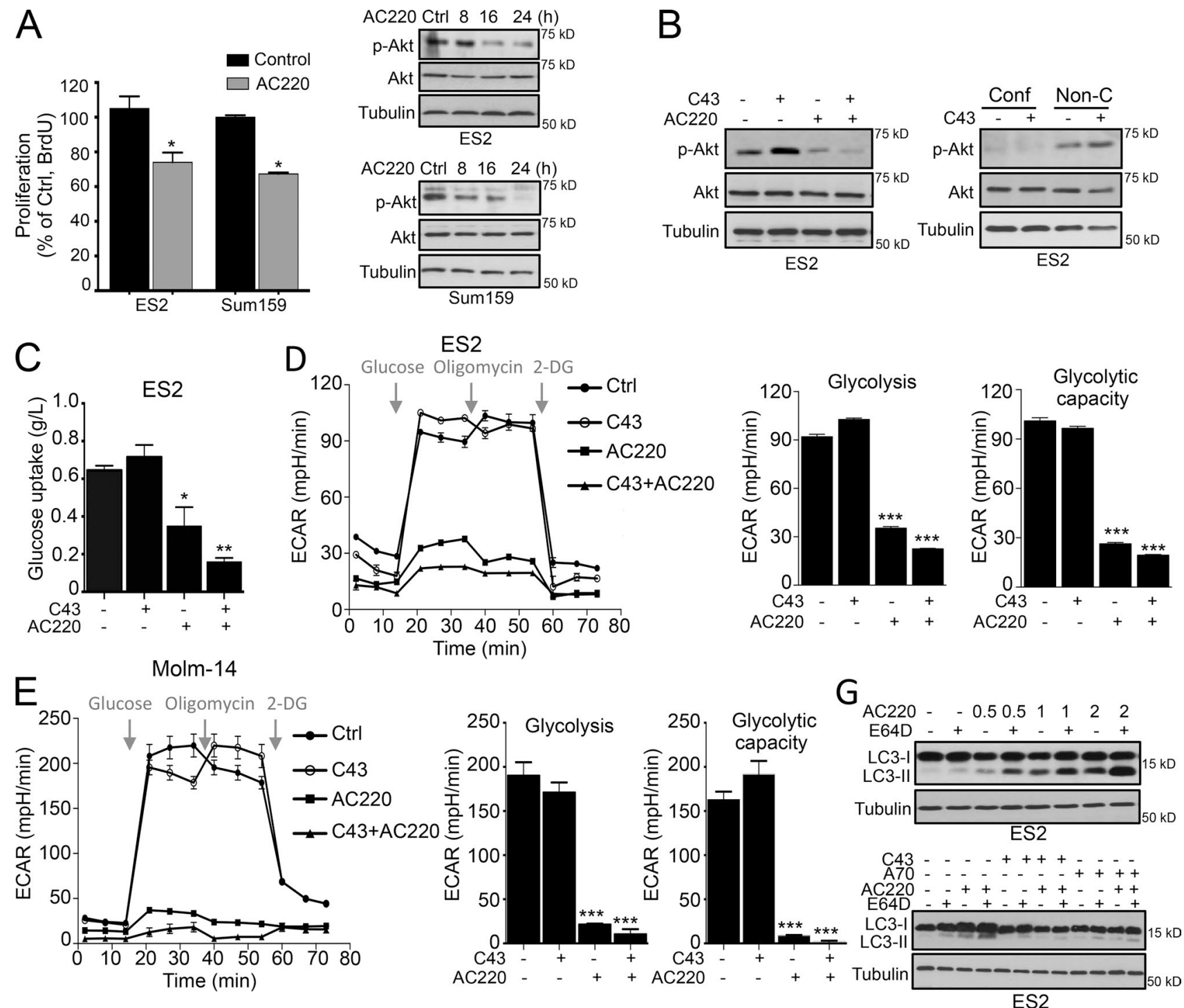

$\mathrm{F}$

ES2

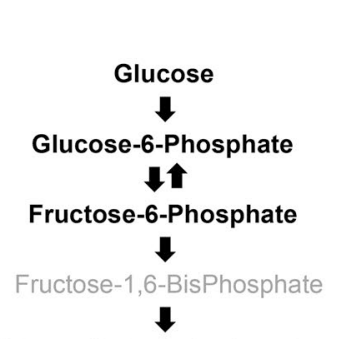

Glyceraldehyde-3-phosphate

$$
\checkmark
$$

3-Phosphoglycerate

11

2-Phosphoglycerate

11

Phosphoenolpyruvate

$\downarrow$

Pyruvate $\Rightarrow$ Lactate
$[\mathrm{U}-13 \mathrm{C}]$-glucose

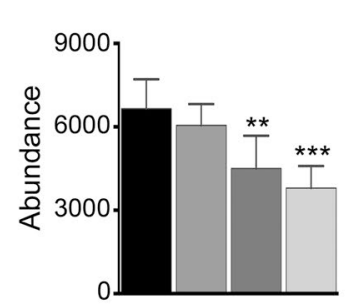

$[\mathrm{U}-13 \mathrm{C}]$-glucose 6-phosphate/ [U-13C]-dihydroxyacetone phosphate
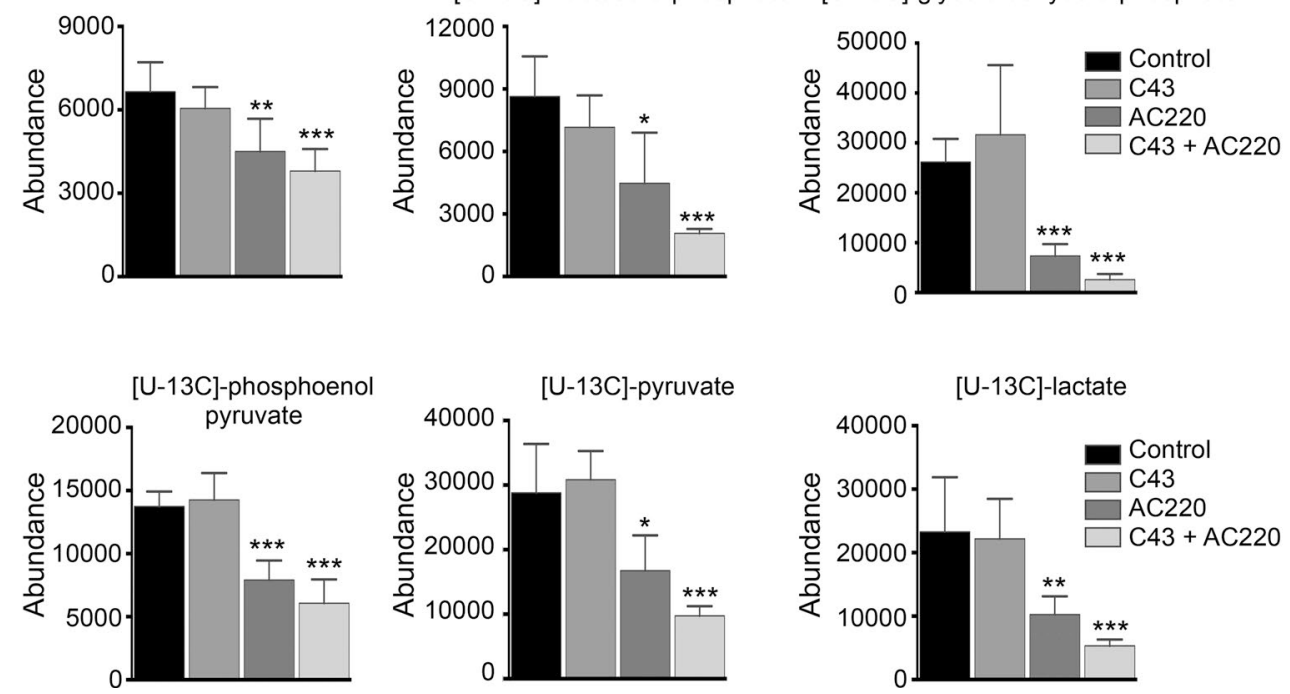

Figure 2. Treatment with AC220 (Quizartinib) reduces glycolysis and induces macroautophagy. (A) Proliferation capacity (\%) of ES2 and Sum 159 cells treated with AC220 for $16 \mathrm{~h}$. Phospho- and total Akt levels of ES2 and Sum 159 cells treated with AC220 up to $24 \mathrm{~h}$. (B) WB of phospho- and total Akt levels of ES2 cells treated with AC220 and C43 (left), or C43 alone in confluent (Conf) or nonconfluent (Non-C) conditions (right) for $24 \mathrm{~h}$. (C) Relative change 
nation treatment led to a significant reduction of cellular ATP levels as well as increased Annexin $\mathrm{V}$ positivity and propidium iodide (PI) uptake (Fig. 3, F and G). This was not inhibited by either zVAD.fmk, a pan caspase inhibitor, or 7-Cl-O-Nec-1 (7N-1), a RIPK1 inhibitor (Degterev et al., 2005). Moreover, no caspase-3 or PARP cleavage was detectable after C43 and AC220 combination treatment, in contrast to the treatment of staurosporine (STS; Fig. 3 G). Instead, siRNA-mediated knockdown of Hsc70 or Lamp2A significantly suppressed the cell death (Fig. $3 \mathrm{H}$ ). These data suggest that the CMA pathway, at least in part, mediates the cell death observed after C43 and AC220 combination treatment.

We observed a major drop in the levels of cellular ATP upon CMA activation induced by combination treatment of AC220 and C43 (Fig. 4 A). The metabolic dysfunction upon AC220 and C43 treatment was also indicated by the significantly elevated cellular ratios of ADP/ATP and NAD+/NADH (Fig. 4, B and C). This prompted us to perform additional metabolic analysis. Notably, we found that single treatment with AC220, which reduced glycolysis (Fig. 2 D), did not drastically affect cellular ATP levels (Fig. 4 B) or mitochondrial oxygen consumption rates (OCRs; Fig. 4 D). Thus, single treatment with either AC220 or C43 had a minimal effect on the cellular ATP levels (Fig. 4 B). Most cells demonstrate plasticity in their ability to generate ATP from either glycolysis or mitochondrial respiration in response to altered cellular metabolism, whereby blockage of one metabolic pathway rapidly shifts cells to generate ATP from the other. Consistently, we found that glutamine uptake was stimulated by AC220 treatment (Fig. 4 E), which might provide an alternative energy source for maintaining cellular ATP levels. Furthermore, AC220 showed no synergy with inhibitors of fatty acid oxidation (FAO), such as Etomoxir or T0070907, but synergized with oligomycin or rotenone, which disrupt mitochondrial respiration (Fig. 4 F). Thus, cancer cells treated with AC220 are in metabolic stress due to inhibition of glycolysis but survive because of concomitant sustained oxidative phosphorylation and autophagy activation. Strikingly, the combination treatment of AC220 and C43 abolished the compensatory metabolism and inhibited glutamine uptake (Fig. 4 E). Therefore, we reasoned that the significant effect of combination treatment on metabolism is due to the concerted actions of both compounds, which induce a rapid collapse in ATP level and lead to cell death via metabolic catastrophe.

Consistent with the importance of CMA in cell death induced by AC220 and C43, measurement of the mitochondrial OCRs of intact cultured cells after combination treatment revealed a significant reduction in the ATP-coupled OCR, which was dramatically rescued in cells with a reduced CMA capacity after siRNA-mediated knockdown of Hsc70 or Lamp-2A (Fig. 4 G). Hsc70 siRNA-mediated rescue of ATP levels was abolished by sublethal concentrations of oligomycin, supporting the role of mitochondrial respiration (Fig. S3 D). Furthermore, siRNA-mediated knockdown of Hsc70 or Lamp2A significantly reversed the ATP levels under combination treatment (Fig. 4 A). These results suggest that CMA may be ac- tivated to mediate cell death after depriving cells of multiple cellular bioenergy sources to lead to a metabolic catastrophe with the combination of AC220 and spautins. Our findings further suggest that a combinatorial inhibition of both FLT3 and autophagy provides a method to effectively inhibit adapted metabolism in cancer cells through the activation of CMA.

\section{CMA promotes the degradation of HK2 and leads to metabolic catastrophe}

To gain further insight into the role of CMA in the death of cancer cells, we performed an unbiased quantitative proteomic approach to characterize the proteins that are specifically degraded via the CMA pathway. To eliminate the contribution from the proteasome, the samples were treated with MG132, which had no effect on the degradation of mutant p53 (Fig. 5 A). We used tandem mass tag (TMT)-based quantitative mass spectrometry to analyze protein level changes in the cytosolic fractions of ES2 cells treated with a combination of C43 and AC220. From 2,051 proteins analyzed, we identified 223 proteins with at least $>1.2$-fold decrease in protein levels after combination treatment, of which 47 proteins exhibited statistically significant reductions $(\mathrm{P}<0.05$; Fig. $5 \mathrm{~B})$. Among these 47 potential CMA substrates, there was a significant enrichment (48\%) of proteins containing one or more pentapeptide motifs that are biochemically related to the CMA-targeting motif (KFERQ). The CMA-targeting motif is degenerate and allows a series of amino acid combinations (Cuervo, 2010), and, consistently, an analysis with relaxed stringency revealed that almost $79 \%$ of all statistically decreased proteins had potential a CMA targeting motif, including p53 and Hsc70, which have been previously reported as bona fide CMA substrates (Fig. 5 B and Table S1).

We verified a subset of this CMA-degradome, including FAM3C, STG1 (hECD), and HK2, which showed a time-dependent reduction upon treatment with a combination of AC220 and C43 (Fig. 5 C). Pretreatment with ClQ, a lysosomal inhibitor, significantly attenuated FAM3C, STG1, and HK2 degradation under the same conditions, whereas the proteasome inhibitor MG132 showed no effect (Fig. 5 D). These results are similar to known CMA substrates such as GAPDH and mutant p53 (Figs. $5 \mathrm{~A}$ and 3 D). Of particular interest to this study were the potential CMA substrates that might be involved in the metabolism and survival of cancer cells. We directed our focus on HK2, a key mediator of metabolism often overexpressed in cancer cells (Wolf et al., 2011; Dey et al., 2012; Patra et al., 2013) and found to be degraded after inhibition of tripeptidyl peptidase II (TPPII), which leads to activation of lysosomal degradation (Lu et al., 2014). No decrease in the mRNA levels of HK2 was found after combination treatment with C43 and AC220 (Fig. S3 E), which verifies that the reduction in the levels of HK2 is due to an increase in degradation. The degradation of HK2 levels was also confirmed using Sum159 cells upon treatment of AC220 and C43 (Fig. S3 F).

HK2 is known as an oncogenic kinase that promotes multiple metabolic pathways that are essential to prevent metabolic stress. HK2 overexpression helps cancer cells maintain growth factor-in-

in glucose levels in the culture medium of ES2 cells treated with AC220 and/or C43 (normalized to cell numbers) for $16 \mathrm{~h}$. (D and E) The glycolytic activity and maximum glycolytic capacity of ES2 (D) or Molm-14 (E) cells, determined by ECAR, after AC220 and C43 treatment for 12 h (ES2) or $8 \mathrm{~h}$ (Molm-14). (F) Glucose flux analysis using $\left[\mathrm{U}^{13} \mathrm{C}\right]$ glucose. A schematic depiction of intermediary metabolites of glycolysis is shown. ${ }^{13} \mathrm{C}$ enrichment of intracellular glucose-derived metabolites, marked in bold, is presented. (G) WB of LC3 protein levels in ES2 cells, treated with increasing concentrations of AC220 and/ or $5 \mu M$ E64D for $16 \mathrm{~h}$ (top), or treated with C43 or A70 in combination with AC220 and/or $5 \mu M$ E64D for $24 \mathrm{~h}$. Anti- $\alpha$-tubulin was used as a loading control. Cells were treated with 0.1\% DMSO (control: vehicle), $1 \mu M$ AC220, $10 \mu M$ C43, or $1 \mu M$ A70, unless otherwise stated. In all the experiments, treatment groups were compared with the control group, unless otherwise shown. Error bars indicate $\pm \mathrm{SD} .{ }^{*}, \mathrm{P}<0.05 ; * *, \mathrm{P}<0.01 ; * * *, \mathrm{P}<0.001$. 
A

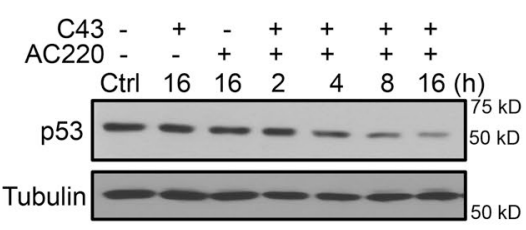

ES2

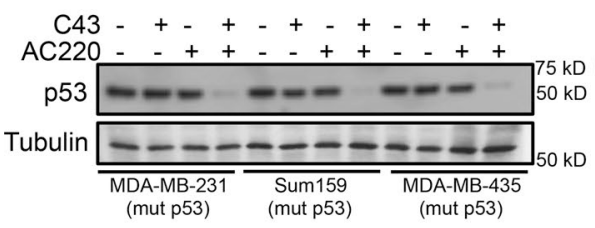

(mut p53)
B

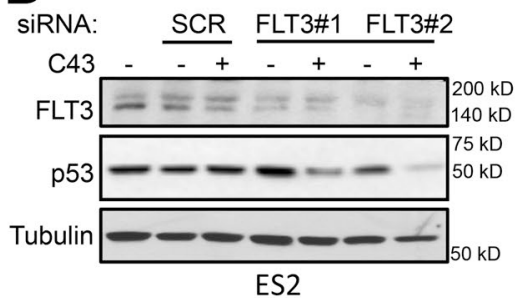

$\mathcal{C}$

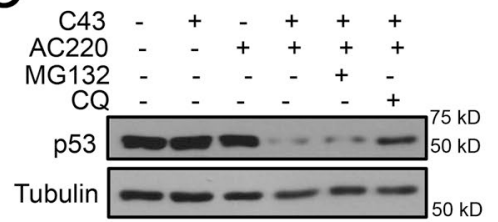

E

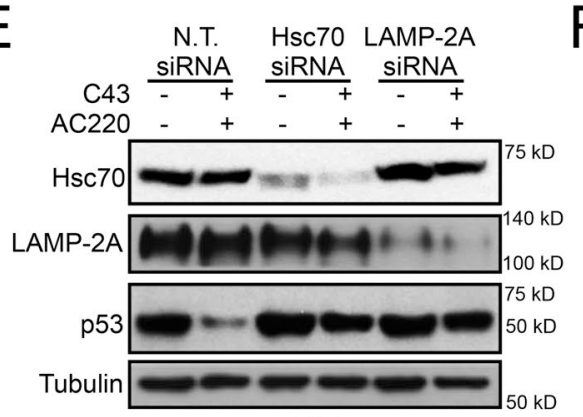

ES2
$\mathrm{F}$

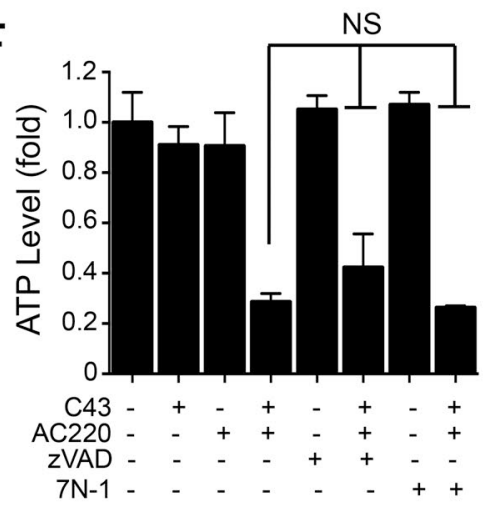

D
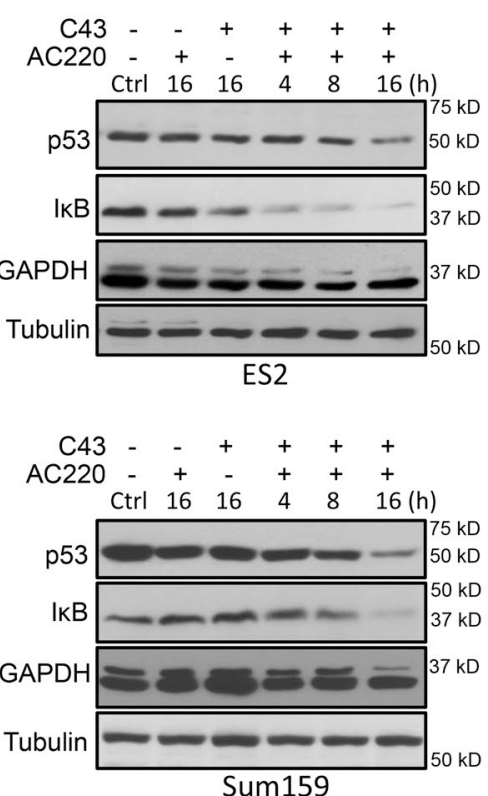

$\mathrm{H}$

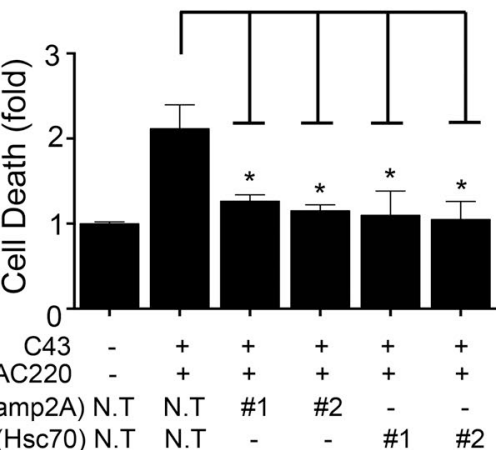

Figure 3. Combination treatment of AC220 and spautins induces CMA. (A) WB quantification of mutant p53 levels in ES2 (top) for the indicated time points and in MDA-MB-231, Sum 159, and MDA-MB-435 (bottom) cells treated with AC220 and/or C43 for 16 h. (B) Mutant p53 levels in scramble (SCR) or FLT3 siRNA-transfected ES2 cells treated with C43 for $24 \mathrm{~h}$. This experiment used the same blot as that in Fig. $1 \mathrm{E}$ with additional antibodies. (C) p53 levels in ES2 cells treated with C43 and AC220 for $24 \mathrm{~h}$ in the absence or presence of proteasome (MG 132) or lysosomal inhibitor (ClQ). (D) p53, IKB, and GAPDH levels in ES2 and Sum 159 cells treated with AC220 and/or C43 for the indicated time points. (E) WB of p53, Lamp2A, and Hsc70 levels in nontargeting (N.T.), Hsc70, or Lamp2A siRNA-transfected ES2 cells treated with AC220 and/or C43 for $24 \mathrm{~h}$. (F) The cellular ATP levels (fold) in ES2 cells treated with AC220 and/or C43 in the presence or absence of zVAD or 7N-1 for $16 \mathrm{~h}$. (G) Cell death indicated as Annexin V/Pl positivity (fold) and WB of PARP-1 and caspase-3 cleavage in ES2 cells treated with AC220 and/or C43 in the presence or absence of zVAD or 7N-1 for $24 \mathrm{~h}$. STS was used as a positive cell death inducer. $(\mathrm{H})$ Cell death (fold) of nontargeting (N.T.), Lamp2A, or Hsc70 siRNA-transfected ES2 cells treated with AC220 and/or C43 for 24 h. Anti- $\alpha$-tubulin was used as a loading control. Cells were treated with $0.1 \%$ DMSO (control: vehicle) or $1 \mu M$ AC220 and $10 \mu M C 43$, unless otherwise stated. In all the experiments, treatment groups were compared with the control group, unless otherwise indicated. Error bars indicate $\pm \mathrm{SD} .{ }^{*}, \mathrm{P}<0.05 ;{ }^{* *}, \mathrm{P}<0.01$.

dependent glucose metabolism to suppress cell death after growth factor withdrawal (Mason et al., 2010); thus, its degradation by CMA might be causal to the observed metabolic malfunction upon combination treatment. We postulated that HK2 degradation upon combination treatment, when glycolysis is blocked, might lead to metabolic catastrophe and cell death. Consistently, knockdown of
HK2, but not of p53, SGT1, or FAM3C, in ES2 cells significantly induced cell death (Fig. $5 \mathrm{E}$ and S3 G), confirming that the loss of HK2 alone is sufficient to induce cell death.

Interestingly, the potential CMA-targeting motif we identified in $\mathrm{HK} 2$ is ${ }_{712} \mathrm{QRFEK}_{716}$, a stringent CMA motif, and is involved in direct binding to glucose molecules (Fig. 5 F; Mu- 
A

B
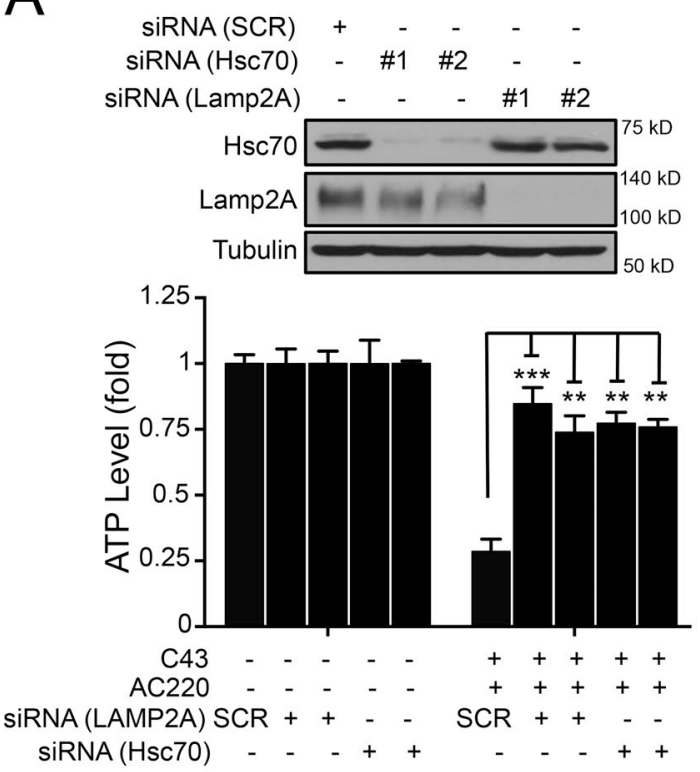

$\mathrm{F}$

DMSO Ctrl $\square$ Oligomycin $\square$ Rotenone $\square$ Etomoxir

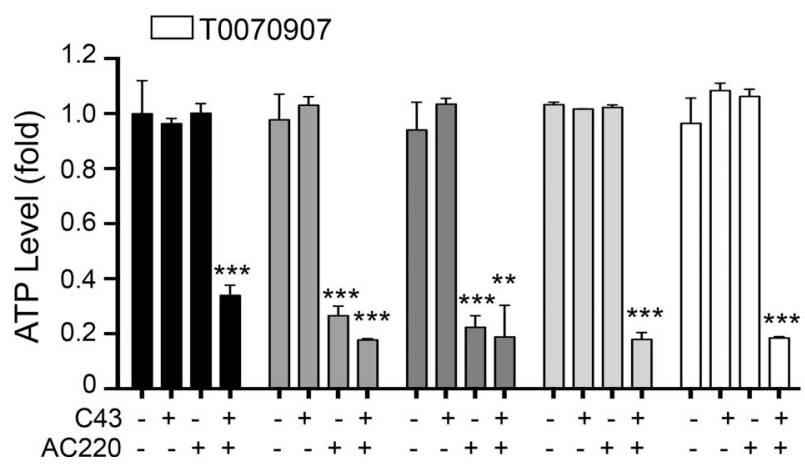

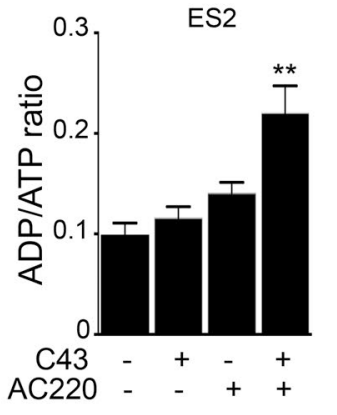

ES2

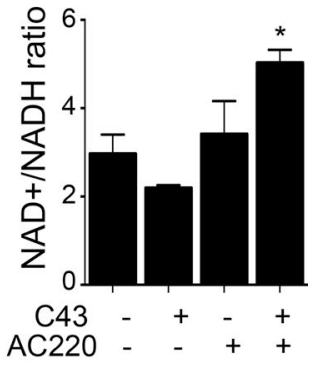

E

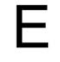

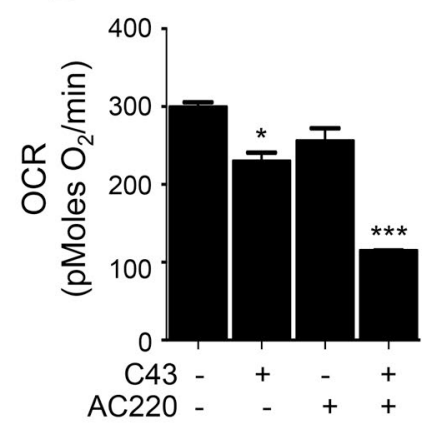
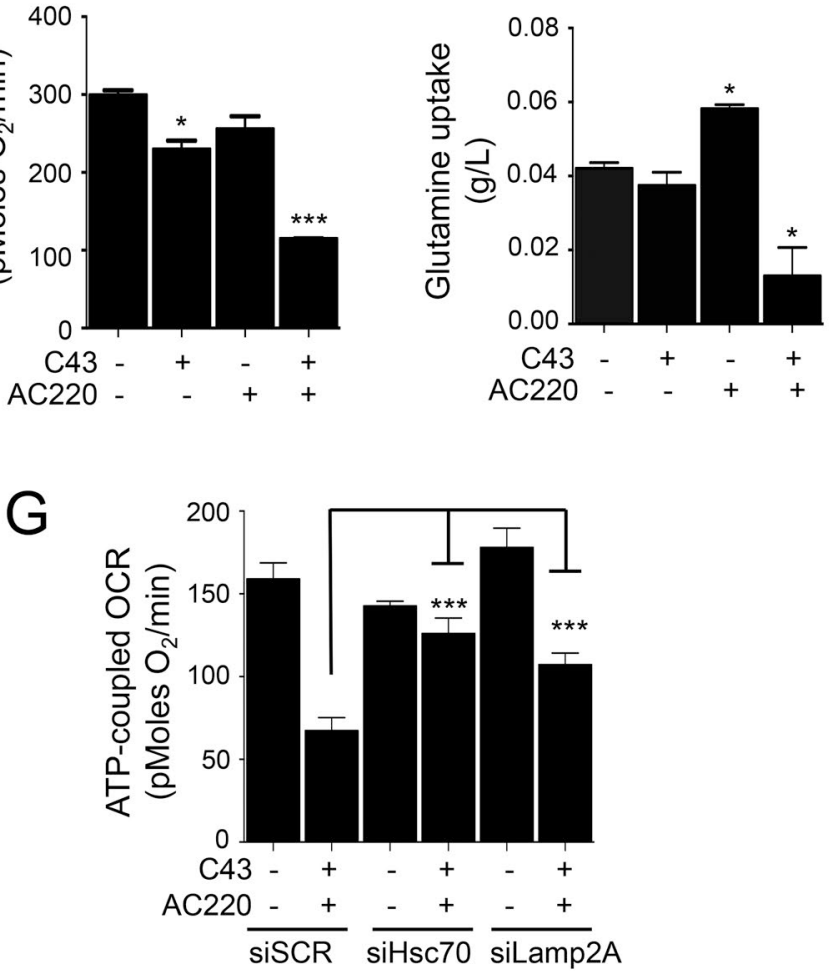

Figure 4. Combination treatment of AC220 and spautins induces metabolic catastrophe. (A) The cellular ATP levels (fold) in scramble (SCR), Lamp2A, or Hsc70 siRNA-transfected ES2 cells treated with AC220 and/or C43 for 16 h (C43). WB shows the Hsc70 and Lamp2A siRNA knockdown efficiencies. (B) The intracellular ATP content and ADP/ATP ratio of ES2 cells treated with AC220 and/or C43 for $12 \mathrm{~h}$. (C) Relative change in the NAD+/NADH ratio in lysates from ES2 cells treated with AC220 and/or C43 for $12 \mathrm{~h}$. (D) The rate of mitochondrial respiration measured by OCR in ES2 cells treated with AC220 and/or C43 for $8 \mathrm{~h}$. (E) Relative change in glutamine levels in the culture medium of ES2 cells treated with AC220 and/or C43 (normalized to cell numbers) for $16 \mathrm{~h}$. (F) The cellular ATP levels in ES2 treated with AC220 and/or C43 in the absence or presence of Oligomycin, Rotenone, Etomoxir, or T0070907 for 16 h. (G) ATP-coupled OCR in scramble (SCR), Hsc70, or Lamp2A siRNA-transfected ES2 treated with AC220 and/or C43 for 8 h. Anti- $\alpha$-tubulin was used as a loading control. Cells were treated with $0.1 \%$ DMSO (control: vehicle) or $1 \mu M$ AC220 and $10 \mu M$ C43, unless otherwise stated. In all the experiments, treatment groups were compared with the control group, unless otherwise shown. Error bars indicate \pm SD. ${ }^{*}, \mathrm{P}<0.05$; $* *, \mathrm{P}<0.01 ; * * *, \mathrm{P}<0.001$

lichak et al., 1998). In the presence of glucose, the CMA motif is buried in the structure after being bound to glucose. In the absence of glucose, the CMA motif of HK2 is likely exposed, accessible to recognition and binding with Hsc70, and thereby Lamp2A. Accordingly, upon C43 and AC220 treatment, when cellular glucose levels are extremely low, the binding of HK2 with both Hsc70 and Lamp2A is enhanced as shown by coimmunoprecipitation experiments (Fig. 5 F). Consistently, treatment of cells with 2-deoxy-D-glucose (2DG), which completely inhibits glucose metabolism, leads to HK2 degradation and cell death in combination with C43 (Fig. S3 H). Furthermore, siRNAmediated knockdown of either Hsc70 or Lamp2A before the combination treatment rescued HK2 depletion (Fig. $5 \mathrm{G}$ and S3 I), which suggests that the degradation of HK2 is mediated through the activation of the CMA pathway.

To verify the degradation of HK2 in the lysosomes, we isolated lysosomal fractions of cells treated with C43 and AC220. After combination treatment, the lysosomal fractions of ES2 cells showed a marked enrichment of HK2 (Fig. $5 \mathrm{H}$ ), showing the lysosomal targeting of HK2 upon CMA activation. To functionally 
A

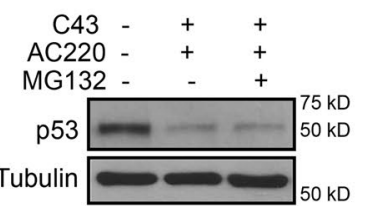

C

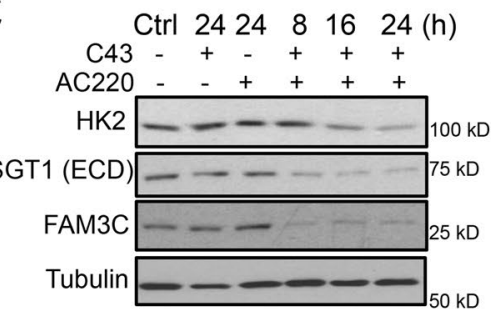

D
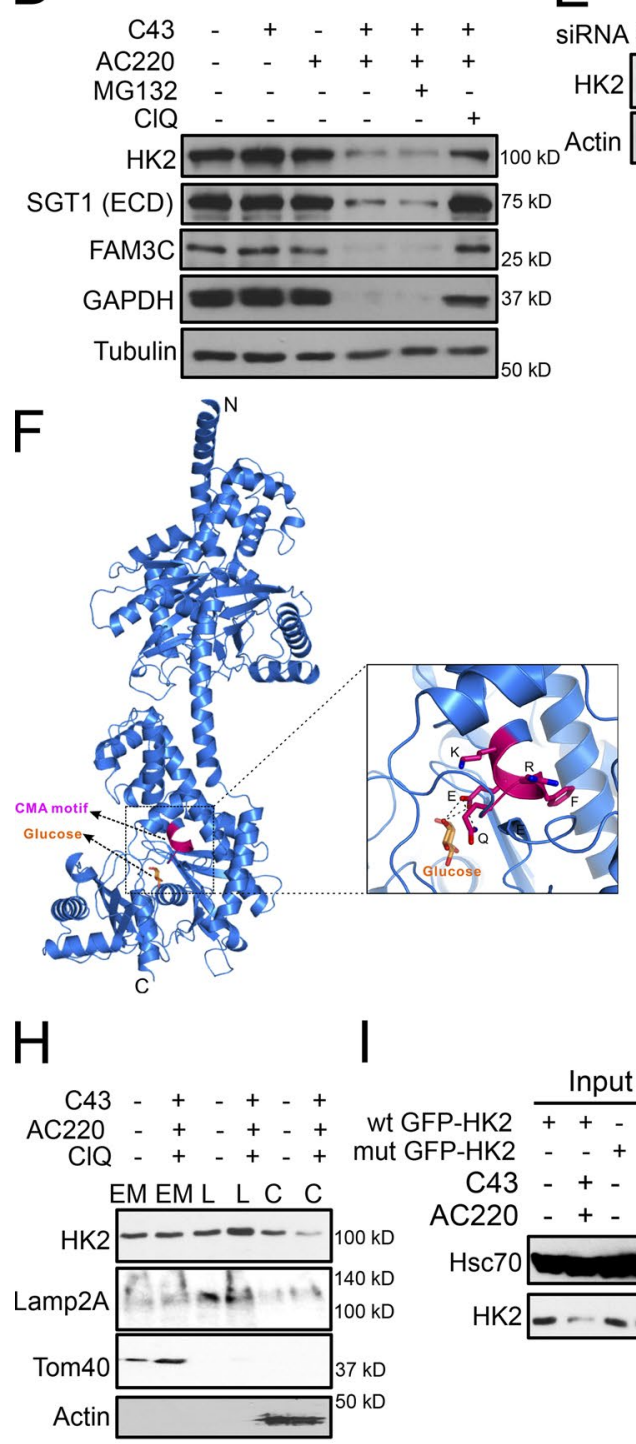

$E$
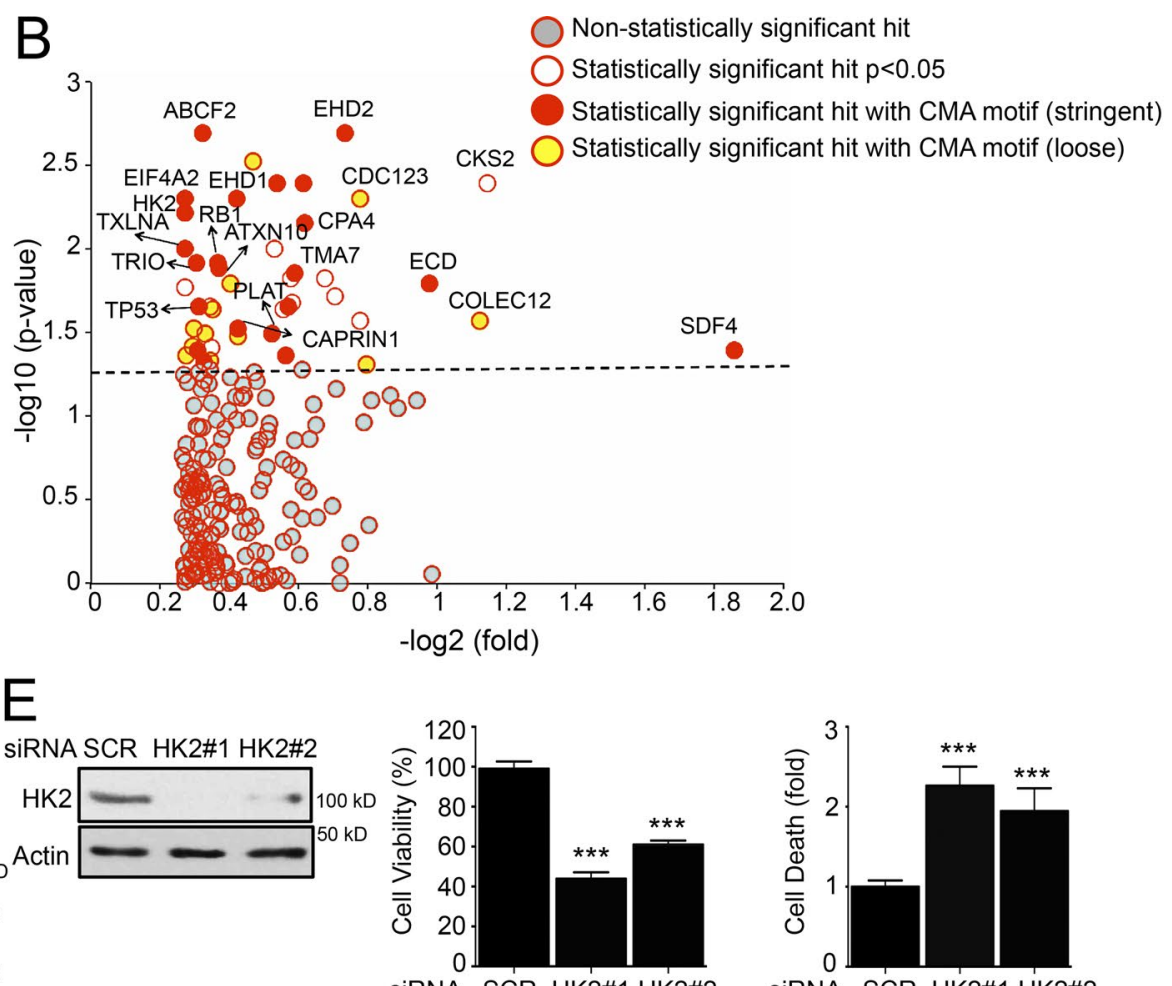

SiRNA SCR HK2\#1 HK2\#2

SiRNA SCR HK2\#1 HK2\#2

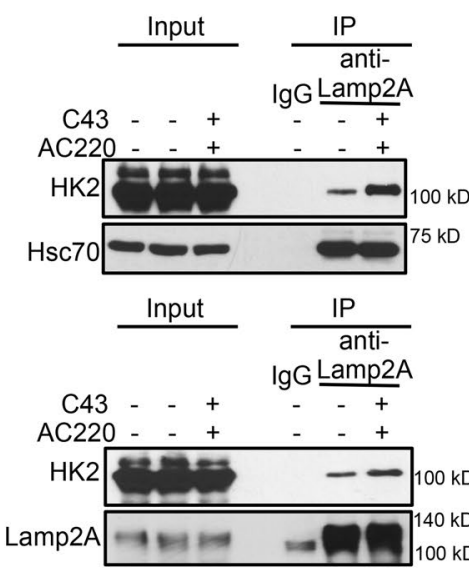

G siRna N.T N.T Hsc70 Hsc70

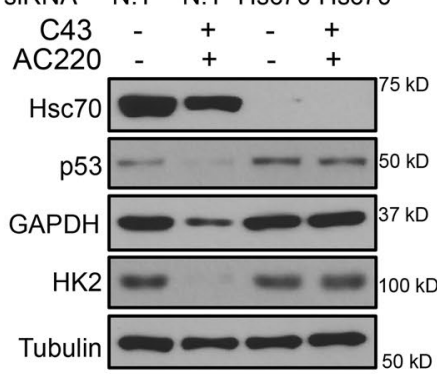

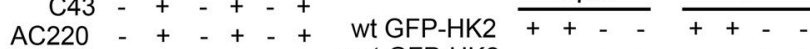

$J$

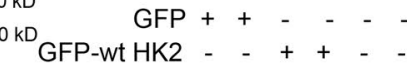

GFP-mut HK2 - - - + +

$\mathrm{C} 43+\mathrm{AC} 220++\ldots+$

HK2 ‥ $100 \mathrm{kD}$

Tubulin $-\infty-\infty-\infty$

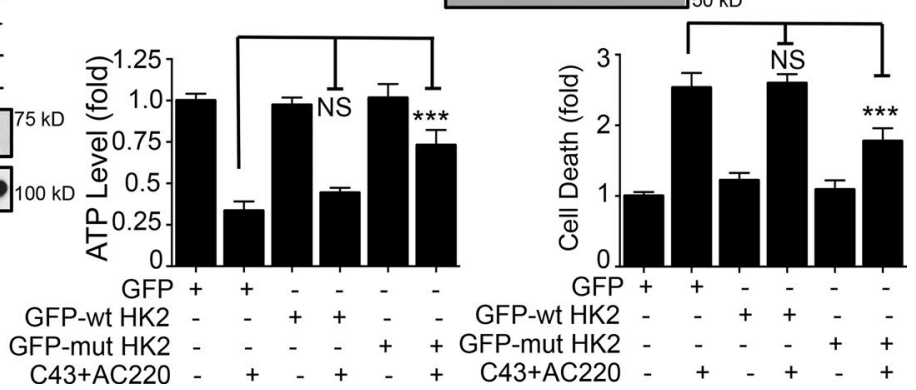

Figure 5. Degradation of HK2 via CMA leads to metabolic stress and cell death. (A) Mutant p53 levels in the cytosolic fraction of ES2 cells treated with AC220 and for $16 \mathrm{~h}$ in the absence or presence of MG132. (B) Scatter plot depicting proteins identified and quantified in a quantitative proteomics experiment. Proteins significantly enriched as containing one or more stringent or loose motif biochemically related to KFERQ are highlighted as potential 
determine the importance of the identified ${ }_{712} \mathrm{QRFEK}_{716}$ motif in HK2 for its degradation induced by the combination treatment, we generated an HK2 mutant (Q712A;R713A) with a two-amino-acid mutation in its CMA motif ${ }_{712} \mathrm{AAFEK}_{716}$. We found that the binding of Q712A;R713A mutant HK2 protein with Hsc70 in 293T cells with combination treatment was significantly reduced (Fig. 5 I). The degradation of Q712A;R713A mutant HK2 in ES2 cells treated with $\mathrm{C} 43$ and AC220 was markedly repressed compared with that of wild-type (wt) HK2 (Fig. 5 J, top). Finally, we found that the gain of function of the CMA-resistant HK2 mutant was to rescue the observed ATP drop and cell death effects induced by the combination treatment of AC220 and C43 (Fig. 5 J, bottom). From these results, we conclude that HK2 is a CMA substrate with a canonical CMA motif whose accessibility is regulated by the presence of glucose and that the degradation of HK2 through CMA may lead to metabolic catastrophe and cell death.

Our study suggests that the degradation of metabolic proteins through the activation of the CMA pathway might directly connect metabolic malfunction/collapse to cell death, providing a molecular link between metabolic stress and cell death. In addition, because HK2 is known to be tightly associated with the mitochondrial membrane via interaction with the mitochondrial voltage-dependent anion channel (Kroemer and Pouyssegur, 2008), the loss of HK2 might not only block glucose metabolism but also directly impair the health of mitochondria. We propose that pharmacological manipulation of the FLT3 pathway and autophagy may lead to the activation of CMA pathway in cancer cells and a mechanism to promote cancer cell death, even of those that have acquired mutations to overcome intrinsic apoptosis, through metabolic catastrophe. Our results support the proposal of a model in which CMA may be exploited as a method to eliminate cancer cells by activating the degradation of the pro-oncogenic proteins, such as HK2. The ability of CMA to promote the degradation of HK2 and mutant p53 suggests the potential utility of CMA in manipulating cellular metabolism as a means of novel anticancer therapeutics.

\section{Materials and methods}

\section{Cell lines and culture conditions}

Cell lines were cultured as described previously (Liu et al., 2011; Vakifahmetoglu-Norberg et al., 2013). The AML, HEL, OCI-AML3, and Molm-14 cell lines were cultured in RPMI (10\% FBS and 1\% penicillin/streptomycin). For glucose-free conditions, cell culture media with no glucose was supplemented with dialyzed FBS. Cells were treated in nonconfluent condition ( $\sim 60 \%$ confluence) or in contact-inhibited $100 \%$ confluent condition. Cell proliferation measurement was per- formed using the BrdU Cell Proliferation Assay kit (Cell Signaling Technology) according to the manufacturer's protocol.

\section{Chemical compounds and treatments}

C43 $(10 \mu \mathrm{M})$, AC220 $(1 \mu \mathrm{M})$, or A70 $(1 \mu \mathrm{M})$ were used unless otherwise stated. STS $(1 \mu \mathrm{M})$, MG132 $(10 \mu \mathrm{M})$, ClQ $(50 \mu \mathrm{M})$, LY294002 (10 $\mu \mathrm{M})$, GDC-0941 (0.5 $\mu \mathrm{M})$, MK2206 $(1 \mu \mathrm{M})$, oligomycin $(0.1 \mu \mathrm{M})$, Rotenone $(0.1 \mu \mathrm{M})$, Etomoxir $(5 \mu \mathrm{M})$, T0070907 $(5 \mu \mathrm{M})$, D-glucose (11 $\mathrm{mM}), 2 \mathrm{DG}(1,2$ or $5 \mathrm{mM})$, zVAD.fmk $(20 \mu \mathrm{M}), 7 \mathrm{~N}-1(20 \mu \mathrm{M})$, and doxorubicin $(1.25 \mu \mathrm{g} / \mathrm{ml})$ were also used. DMSO (0.1\%) was used as a control. Cell viability and ATP levels were measured using a CellTiterGlo ATP assay (Promega). The ADP/ATP and NAD+/NAHD ratios were determined with assay kits (Abcam) according to the manufacturer's instructions. Annexin V/PI staining was detected by using a flow cytometer using an FITC Annexin V Apoptosis Detection kit I (BD). Cell death/survival was measured using the ToxiLight bioassay (Thermo Fisher Scientific). Cellular fractionation was performed using differential centrifugation and hypotonic lysis of lysosomes, according to Schröter et al. (1999). Coimmunoprecipitation was performed using RIPA buffer and $1 \mu \mathrm{g}$ antibody per sample incubated with $\mathrm{A} / \mathrm{G}$ for 2 h. For genetic knockdown experiments, scramble (SCR)/nontargeting (N.T) or siRNA targeting ATG7 (GE Healthcare) Lamp2A, Hsc70 (GE Healthcare), FLT3 (Santa Cruz Biotechnology, Inc.), or HK2, p53, SGT1, and FAM3C (GenePharma) were used at $40 \mathrm{nM}$. siRNA efficiency was monitored $48-72 \mathrm{~h}$ after transfection.

\section{Small molecule compound screen}

ES2 cells seeded in nonconfluent conditions in a 384-well plate were exposed to 8,248 small molecules from the ICCB known bioactive chemical library. A70 was used at $100 \mathrm{nM}$. For all plates, doxorubicin [1.25 $\mu \mathrm{g} / \mathrm{ml}]$ was used as a positive control for cell death. Plates were screened in duplicate. After $30 \mathrm{~h}$ of treatment time, the CellTiter-Glo Luminescent Cell Viability assay was performed. Possible hits were based on $\mathrm{z}$ scores calculated using the formula $\mathrm{Z}=\left(\mathrm{X}-\mathrm{Ave} \_\mathrm{Neg}\right) /$ SD_Neg or $25 \%$ of difference in cell survival between library alone and in combination with A70. 115 compounds were identified and cherry-picked for a secondary screen.

\section{Cellular metabolic measurements}

ECAR and OCR were measured in real time using the Seahorse XFp analyzer instrument according to the Seahorse standard protocols: XFp Glycolysis Stress Test kit (http://www.seahorsebio.com/resources/ pdfs/manual-xf96-glycolysis-stress-test-kit.pdf) and XFp Cell Mito Stress Test kit (http://www.seahorsebio.com/resources/pdfs/manualxf24-cell-mito-stress-test-kit.pdf).

Glucose and lactate were measured in spent medium using a multiparameter bioanalytical system (YSI 7100; Yellow Springs Instruments). Glucose uptake is presented as the net decrease in concentra-

CMA substrates. (C) HK2, SGT1, and FAM3C protein levels in ES2 cells treated with AC220 and C43 up to $24 \mathrm{~h}$. (D) WB analysis of HK2, SGT1, FAM3C, p53, and GAPDH levels in ES2 cells treated with AC220 and C43 for $16 \mathrm{~h}$ in the absence or presence of proteasome (MG132) or lysosomal inhibitor (ClQ). This experiment (bottom row) used the same blot as in the bottom row of Fig. $3 \mathrm{C}$ with additional antibodies. (E) Cell viability (\%) and cell death (fold) of scramble (SCR) or HK2 siRNA-transfected ES2 cells at $72 \mathrm{~h}$ after transfection. (F) The combined ribbon representation and stick model showing the overall structure and the CMA motif of HK2 protein in complex with glucose. The interaction of HK2 with Hsc70 or Lamp2A, after AC220 and/or C43 treatment, was analyzed by coimmunoprecipitation. (G) WB of HK2, p53, and GAPDH levels in ES2 cells transfected with nontargeting (N.T) or $\mathrm{Hsc} 70$ siRNA, treated with AC220 and C43 for $12 \mathrm{~h}$. (H) The localization of HK2 in cellular endosomal/mitochondria (EM), lysosomal (L), or cytosolic (C) fractions in ES2 cells treated with $A C 220$ and $C 43$ for $16 \mathrm{~h}$, in the presence of the lysosomal inhibitor (CIQ). Lamp2A, Tom 40, or $\beta$-actin were used as markers for the fractions. (I) The interaction of wt or Q712A;R713A mutant GFP-HK2 with Hsc70, after AC220 and/or C43 treatment, analyzed in 293T cells by coimmunoprecipitation. (J) WB analysis, cell viability (\%), and cell death (fold) of wt and Q712A;R713A mutant GPF-HK2-expressing ES2 cells after AC220 and C43 treatment for $16 \mathrm{~h}$. Anti- $\alpha$-tubulin was used as a loading control. Cells were treated with $0.1 \%$ DMSO (control: vehicle) or $1 \mu M$ AC220 and $10 \mu \mathrm{M} \mathrm{C43,} \mathrm{unless} \mathrm{otherwise} \mathrm{stated.} \mathrm{In} \mathrm{all} \mathrm{the} \mathrm{experiments,} \mathrm{treatment} \mathrm{groups} \mathrm{were} \mathrm{compared} \mathrm{with} \mathrm{the} \mathrm{control} \mathrm{group,} \mathrm{unless} \mathrm{otherwise} \mathrm{shown.}$ Error bars indicate $\pm \mathrm{SD}$. ***, $\mathrm{P}<0.001$ 
tion, and lactate production as the net increase in concentration after normalization to cell numbers.

\section{Metabolite extraction and flux analysis}

ES2 cell samples treated for $8 \mathrm{~h}$ were incubated with $\left[\mathrm{U}^{13} \mathrm{C}_{6}\right]$ glucose and DMSO, $1 \mu \mathrm{M} \mathrm{AC} 220,10 \mu \mathrm{M} \mathrm{C} 43$, and $1 \mu \mathrm{M} \mathrm{AC} 220+10 \mu \mathrm{M} \mathrm{C} 43$ for an additional $6 \mathrm{~h}$. Metabolites were extracted using $\mathrm{MeOH} / \mathrm{ACN} / \mathrm{H}_{2} \mathrm{O}(2: 2: 1$, $\mathrm{vol} / \mathrm{vol})$. The extracts were reconstituted in $\mathrm{ACN} / \mathrm{H}_{2} \mathrm{O}(1: 1$, vol/vol), insoluble debris were removed, and extracts were transferred to HPLC vials for liquid chromatography-mass spectrometry (LC-MS) analysis. The LC-MS analysis was performed using a UPLC system (1290 series; Agilent Technologies) coupled to a Q-TOF mass spectrometer (Agilent 6550 iFunnel Q-TOF LC-MS; Agilent Technologies) for the analyses.

\section{Protein digestion and TMT labeling for mass spectrometry and LC-MS/MS}

Three biological replicates of lysates of ES2 cells treated with C43 and $\mathrm{AC} 220$ for $16 \mathrm{~h}$ (MG132 for the last $4 \mathrm{~h}$ ) were precipitated by methanol/chloroform, and protein samples were resuspended in $8 \mathrm{M}$ urea/100 mM TEAB, pH 8.0, and reduced, then alkylated with $10 \mathrm{mM}$ Tris (2-carboxyethyl) phosphine hydrochloride and $55 \mathrm{mM}$ iodoacetamide, followed by tryosin digestion. The resultant peptides were labeled with 6-plex TMT reagents as the user manual describes (Thermo Fisher Scientific). The resulted 6-plex mixture of peptides was analyzed on a hybrid LTQ-Orbitrap Velos mass spectrometer (Thermo Fisher Scientific) using a modified 12-step MudPIT separation that has been described previously (Washburn et al., 2001; Rauniyar et al., 2015). Application of ms scan functions and HPLC solvent gradients were controlled with the Xcalibur data system (Thermo Fisher Scientific).

\section{Data analysis}

Data were extracted from the Xcalibur data system format into MS1 and MS2 formats using in-house software. The peptides/proteins were identified by the Integrated Proteomics Pipeline-IP2 software (Integrated Proteomics Applications, http://www.integratedproteomics.com/) using ProLuCID and DTASelect2 (Tabb et al., 2002) with a decoy database strategy. The precursor mass tolerance was $50 \mathrm{ppm}$ and the fragment mass tolerance was $20 \mathrm{ppm}$. The precursor delta mass cutoff was $10 \mathrm{ppm}$ in DTASelect. The searches were against Uniprot Human protein database (version 07-05-2014). The ratios of treated versus control samples were calculated to normalized protein intensities. A sample difference of $\mathrm{P}<0.05$ was considered statistically significant (Student's $t$ test). The stringent $([\mathrm{Q} / \mathrm{N}][\mathrm{K} / \mathrm{R}][\mathrm{F} / \mathrm{I} / \mathrm{V} / \mathrm{L}][\mathrm{E} / \mathrm{D}][\mathrm{K} / \mathrm{R}],[\mathrm{K} / \mathrm{R}][\mathrm{F} / \mathrm{I} / \mathrm{V} / \mathrm{L}][\mathrm{E} / \mathrm{D}][\mathrm{K} / \mathrm{R}]$ $[\mathrm{Q} / \mathrm{N}])$ and relaxed $([\mathrm{Q} / \mathrm{N} / \mathrm{V}][\mathrm{D} / \mathrm{E} / \mathrm{K} / \mathrm{R} / \mathrm{F}][\mathrm{E} / \mathrm{K} / \mathrm{V} / \mathrm{L}][\mathrm{D} / \mathrm{E} / \mathrm{K} / \mathrm{R} / \mathrm{V} / \mathrm{F} / \mathrm{L}]$ [D/E/Q]) CMA motifs were designed based on Kaushik and Cuervo (2012) using MotifGen software (http://www.proteinguru.com/toolbox/ motifgen/). Protein sequences were batch retrieved from Uniprot (version 07-05-2014, http://www.uniprot.org/) and CMA motif searches were performed using the regular expression search engine BASALT (http://www.proteinguru.com/toolbox/basalt/).

\footnotetext{
Autophagy index analysis

Image analysis of H4-GFP-LC3 cells fixed with 4\% paraformaldehyde (Sigma-Aldrich) and stained with $3 \mu \mathrm{g} / \mathrm{ml}$ DAPI (Sigma-Aldrich), using ArrayScan HCS 4.0 Reader with a 20× objective lens (Cellomics ArrayScan VTI; Life Technologies). The Spot Detector BioApplication (Thermo Fisher Scientific) was used to acquire and analyze the images after optimization. Images of 1,000 cells for each compound treatment were analyzed to obtain the mean cell number per field, fluorescence spot number, area, and intensity per cell. DMSO and rapamycin were used as negative or positive controls, respectively. The percentages of changes of LC3-GFP were calculated by dividing with that of DMSO-
}

treated samples. The images were also analyzed by using a conventional fluorescence microscope for visual inspection. The experiments were repeated three times with consistent results.

\section{Site-directed mutagenesis}

A site-directed mutagenesis kit (F-541; Thermo Fisher Scientific) was used to generate the $Q 712 A ; R 713 A$ mutant gene and the mutation was verified by DNA sequencing. Mutagenesis primers were designed using QuikChange Primer Design software (www.genomics.agilent.com/primerDesignProgram.jsp): HK2_Q739A+R740A_sense, 5'-CTTTCACTCAACCCCGGCAAGGCGGCGTTCGAGAAAATGATCAGTGG-3'; and HK2_Q739A+R740A_anti-sense, 5'-CCACTGATCATTTTCTCGAACGCCGCCTTGCCGGGGTTGAGTGAAAG-3' .

\section{Real-time quantitative PCR}

RNA was isolated using an RNA isolation RNAqueous kit (Ambion). Reverse-transcribed cDNAs from the samples were used as templates (using Maxima First Strand cDNA synthesis Kit for RT-qPCR). cDNA template was mixed with Maxima SYBR Green qPCR Master Mix (Thermo Fisher Scientific) and amplified using a 7500 Real-Time PCR System (Applied Biosystems) with the following primers: HK2 forward, 5'-CAAAGTGACAGTGGGTGTGG-3'; HK2 reverse, 5'-GCCAGGTCCTTCACTGTCTC-3'; p53 forward, 5'-GTTCCGAGAGCTGAATGAGG-3'; p53 reverse, 5' -TTATGGCGGGAGGTAGACTG-3'; $\beta$-actin forward, $5^{\prime}$-GCAAGCAGGAGTATGACGAG-3'; and $\beta$-actin reverse, 5'-CAAATAAAGCCATGCCAATC-3'.

\section{Statistical analyses}

All graphs were analyzed using GraphPad Prism 6 software. Quantitative data represented by bar graphs from at least three independent experiments, in triplicate, are shown and expressed as mean \pm SD followed by the analysis of variance (Vaux, 2012). Some data are presented as fold change over control (DMSO). In all the experiments, treatment groups were compared with the control group, unless otherwise shown. *, $\mathrm{P}<0.05$; **, $\mathrm{P}<0.01$; ***, $\mathrm{P}<0.001$.

\section{Antibodies used}

Primary antibodies used were as follows: anti-p53 (\#2524; Cell Signaling Technology), anti-USP10 (ab72486; Abcam), anti-USP13 (16840-1-AP; ProteinTech), anti-BECN1 (H-300, sc-11427; Santa Cruz Biotechnology, Inc.), anti-tubulin (T8203; Sigma-Aldrich), anti-FLT3 (\#3462; Cell Signaling Technology), anti-p-FLT3 (\#3466; Cell Signaling Technology), anti-Lamp2A (sc-18822; Santa Cruz Biotechnology, Inc.), anti-Hsc70 (HSPA8; 10654-1-AP; Proteintech), anti-Akt (\#9272; Cell Signaling Technology), anti-p-Akt (\#9271; Cell Signaling Technology), anti-LC3 (L7543; Sigma-Aldrich), anti-PARP (556494; BD), anticleaved caspase-3 (\#9661; Cell Signaling Technology), anti-GAPDH (sc-166545; Santa Cruz Biotechnology, Inc.), anti-Erk (\#9102; Cell Signaling Technology), anti-p-Erk (\#9101; Cell Signaling Technology), anti-IкB (sc-371; Santa Cruz Biotechnology, Inc.), anti-HK2 (\#2867; Cell Signaling Technology), anti-SGT1 (ab99293; Abcam), anti-FAM3C (ab56065; Abcam), anti-Tom40 (sc-11414; Santa Cruz Biotechnology, Inc.), and anti-actin (sc-81178; Santa Cruz Biotechnology, Inc.). Quantifications of the WBs are provided in Tables S2 and S3.

\section{Online supplemental material}

Fig. S1 shows the structure of A70, the autophagy index in response to A70, and data relevant to single or combination treatment of $\mathrm{C} 43$, $\mathrm{A} 70$, or AC220 in different culture conditions, as well as phospho- and total FLT3 protein levels of all cell lines tested in the study. In addition, cell viability of cancer cells treated with Lapatinib or Nilotinib are 
presented. Fig. S2 shows additional data relevant to metabolic changes as well as phospho-, total FLT3, and LC3 protein levels of cancer cell lines treated with AC220, C43, and other indicated inhibitors. In addition, cell death and LC3 protein levels of ES2 cells treated with AC220, MK2206, or GDC-0941 are presented. Fig. S3 shows data on mRNA and protein levels of mutant p53, HK2 in cancer cells treated with $\mathrm{AC} 220$, and/or $\mathrm{C} 43$ in the absence or presence of proteasome or lysosomal inhibitors. The protein levels of HK2, p53, and GAPDH after Lamp2A knockdown in ES2 cells treated with AC220 and C43 are also presented. In addition, cellular ATP levels are shown after Hsc70 knockdown with AC220 and/or C43 treatment in the absence or presence of oligomycin. Further, cell viability of HK2, p53, SGT1, or FAM3C knockdown ES2 cells treated with AC220 and/or C43 as well as ES2 cells treated with 2DG and/or C43 are presented. Table S1 shows a list of proteins identified in quantitative proteomics approach. Table S2 and Table S3 show all WB quantifications presented in the main and supplemental figures. Online supplemental material is available at http://www.jcb.org/cgi/content/full/jcb.201503044/DC1.

\section{Acknowledgements}

This work was supported by grants (to J. Yuan) from the Harvard Accelerator Fund (2009), the Ludwig Center at Harvard, and the Chinese Academy of Sciences. H. Vakifahmetoglu-Norberg was supported in part by a fellowship from the Swedish Research Council (VR), the Swedish Society for Medical Research (SSMF), Åke-Wibergs stiftelse, Stiftelsen Lars Hiertas Minne, and Stiffelsen Längmanska Kulturfonden. X. Han and J.R. Yates were supported in part by the National Heart, Lung, and Blood Institute Proteomics Center Award HHSN268201000035C and RO1 MH067880 and 8P41GM103533-17 (to J.R. Yates). H.-g. Xia was supported in part by a postdoctoral fellowship from the Shanghai Institute of Organic Chemistry, Chinese Academy of Sciences.

The authors declare no competing financial interests.

Submitted: 10 March 2015

Accepted: 23 July 2015

\section{References}

Aniento, F., E. Roche, A.M. Cuervo, and E. Knecht. 1993. Uptake and degradation of glyceraldehyde-3-phosphate dehydrogenase by rat liver lysosomes. J. Biol. Chem. 268:10463-10470.

Correa, R.J., T. Peart, Y.R. Valdes, G.E. DiMattia, and T.G. Shepherd. 2012. Modulation of AKT activity is associated with reversible dormancy in ascites-derived epithelial ovarian cancer spheroids. Carcinogenesis. 33:49-58. http://dx.doi.org/10.1093/carcin/bgr241

Cuervo, A.M. 2010. Chaperone-mediated autophagy: selectivity pays off. Trends Endocrinol. Metab. 21:142-150. http://dx.doi.org/10.1016/j.tem.2009.10.003

Cuervo, A.M., W. Hu, B. Lim, and J.F. Dice. 1998. IкB is a substrate for a selective pathway of lysosomal proteolysis. Mol. Biol. Cell. 9:1995-2010. http://dx.doi.org/10.1091/mbc.9.8.1995

Degterev, A., Z. Huang, M. Boyce, Y. Li, P. Jagtap, N. Mizushima, G.D. Cuny, T.J. Mitchison, M.A. Moskowitz, and J. Yuan. 2005. Chemical inhibitor of nonapoptotic cell death with therapeutic potential for ischemic brain injury. Nat. Chem. Biol. 1:112-119. http://dx.doi.org/10.1038/nchembio711

Dey, P., S. Rachagani, S. Chakraborty, P.K. Singh, X. Zhao, C.B. Gurumurthy, J.M. Anderson, S. Lele, M.A. Hollingsworth, V. Band, and S.K. Batra. 2012. Overexpression of ecdysoneless in pancreatic cancer and its role in oncogenesis by regulating glycolysis. Clin. Cancer Res. 18:6188-6198. http://dx.doi.org/10.1158/1078-0432.CCR-12-1789

Elstrom, R.L., D.E. Bauer, M. Buzzai, R. Karnauskas, M.H. Harris, D.R. Plas, H. Zhuang, R.M. Cinalli, A. Alavi, C.M. Rudin, and C.B. Thompson. 2004. Akt stimulates aerobic glycolysis in cancer cells. Cancer Res. 64:3892-3899. http://dx.doi.org/10.1158/0008-5472.CAN-03-2904
Kaushik, S., and A.M. Cuervo. 2012. Chaperone-mediated autophagy: a unique way to enter the lysosome world. Trends Cell Biol. 22:407-417. http:// dx.doi.org/10.1016/j.tcb.2012.05.006

Kayser, S., and M.J. Levis. 2014. FLT3 tyrosine kinase inhibitors in acute myeloid leukemia: clinical implications and limitations. Leuk. Lymphoma. 55:243-255. http://dx.doi.org/10.3109/10428194.2013.800198

Kroemer, G., and J. Pouyssegur. 2008. Tumor cell metabolism: cancer's Achilles' heel. Cancer Cell. 13:472-482. http://dx.doi.org/10.1016/j.ccr.2008.05.005

Liu, J., H. Xia, M. Kim, L. Xu, Y. Li, L. Zhang, Y. Cai, H.V. Norberg, T. Zhang, T. Furuya, et al. 2011. Beclin1 controls the levels of p53 by regulating the deubiquitination activity of USP10 and USP13. Cell. 147:223-234. http://dx.doi.org/10.1016/j.cell.2011.08.037

Lu, W., Y. Zhang, D.O. McDonald, H. Jing, B. Carroll, N. Robertson, Q. Zhang, H. Griffin, S. Sanderson, J.H. Lakey, et al. 2014. Dual proteolytic pathways govern glycolysis and immune competence. Cell. 159:1578-1590. http://dx.doi.org/10.1016/j.cell.2014.12.001

Mason, E.F., Y. Zhao, P. Goraksha-Hicks, J.L. Coloff, H. Gannon, S.N. Jones, and J.C. Rathmell. 2010. Aerobic glycolysis suppresses p53 activity to provide selective protection from apoptosis upon loss of growth signals or inhibition of BCR-Abl. Cancer Res. 70:8066-8076. http://dx.doi. org/10.1158/0008-5472.CAN-10-0608

Mizushima, N., and M. Komatsu. 2011. Autophagy: renovation of cells and tissues. Cell. 147:728-741. http://dx.doi.org/10.1016/j.cell.2011.10.026

Mulichak, A.M., J.E. Wilson, K. Padmanabhan, and R.M. Garavito. 1998. The structure of mammalian hexokinase-1. Nat. Struct. Biol. 5:555-560. http://dx.doi.org/10.1038/811

Nederlof, R., O. Eerbeek, M.W. Hollmann, R. Southworth, and C.J. Zuurbier. 2014. Targeting hexokinase II to mitochondria to modulate energy metabolism and reduce ischaemia-reperfusion injury in heart. Br. J. Pharmacol. 171:2067-2079. http://dx.doi.org/10.1111/bph.12363

Parcells, B.W., A.K. Ikeda, T. Simms-Waldrip, T.B. Moore, and K.M. Sakamoto. 2006. FMS-like tyrosine kinase 3 in normal hematopoiesis and acute myeloid leukemia. Stem Cells. 24:1174-1184. http://dx.doi.org/10.1634/ stemcells.2005-0519

Patra, K.C., Q. Wang, P.T. Bhaskar, L. Miller, Z. Wang, W. Wheaton, N. Chandel, M. Laakso, W.J. Muller, E.L. Allen, et al. 2013. Hexokinase 2 is required for tumor initiation and maintenance and its systemic deletion is therapeutic in mouse models of cancer. Cancer Cell. 24:213-228. http:// dx.doi.org/10.1016/j.ccr.2013.06.014

Rauniyar, N., K. Subramanian, M. Lavallee-Adam, S. Martinez-Bartolome, W.E. Balch, and J.R. Yates III. 2015. Quantitative proteomics of human fibroblasts with I1061T mutation in Niemann-Pick C1 (NPC1) protein provides insights into the disease pathogenesis. Mol. Cell. Proteomics.:1734. http://dx.doi.org/10.1074/mcp.M114.045609

Schröter, C.J., M. Braun, J. Englert, H. Beck, H. Schmid, and H. Kalbacher. 1999. A rapid method to separate endosomes from lysosomal contents using differential centrifugation and hypotonic lysis of lysosomes. J. Immunol. Methods. 227:161-168. http://dx.doi.org/10.1016/ S0022-1759(99)00079-4

Shaw, R.J., and L.C. Cantley. 2006. Ras, PI(3)K and mTOR signalling controls tumour cell growth. Nature. 441:424-430. http://dx.doi.org/10.1038/ nature 04869

Tabb, D.L., W.H. McDonald, and J.R. Yates III. 2002. DTASelect and Contrast: tools for assembling and comparing protein identifications from shotgun proteomics. J. Proteome Res. 1:21-26. http://dx.doi.org/10.1021/ pr015504q

Vakifahmetoglu-Norberg, H., M. Kim, H.G. Xia, M.P. Iwanicki, D. Ofengeim, J.L. Coloff, L. Pan, T.A. Ince, G. Kroemer, J.S. Brugge, and J. Yuan. 2013. Chaperone-mediated autophagy degrades mutant p53. Genes Dev. 27:1718-1730. http://dx.doi.org/10.1101/gad.220897.113

Vaux, D.L. 2012. Research methods: Know when your numbers are significant. Nature. 492:180-181.

Washburn, M.P., D. Wolters, and J.R. Yates III. 2001. Large-scale analysis of the yeast proteome by multidimensional protein identification technology. Nat. Biotechnol. 19:242-247. http://dx.doi.org/10.1038/85686

White, E. 2012. Deconvoluting the context-dependent role for autophagy in cancer. Nat. Rev. Cancer. 12:401-410. http://dx.doi.org/10.1038/nrc3262

Wolf, A., S. Agnihotri, J. Micallef, J. Mukherjee, N. Sabha, R. Cairns, C. Hawkins, and A. Guha. 2011. Hexokinase 2 is a key mediator of aerobic glycolysis and promotes tumor growth in human glioblastoma multiforme. J. Exp. Med. 208:313-326. http://dx.doi.org/10.1084/jem.20101470

Zarrinkar, P.P., R.N. Gunawardane, M.D. Cramer, M.F. Gardner, D. Brigham, B. Belli, M.W. Karaman, K.W. Pratz, G. Pallares, Q. Chao, et al. 2009. AC220 is a uniquely potent and selective inhibitor of FLT3 for the treatment of acute myeloid leukemia (AML). Blood. 114:2984-2992. http:// dx.doi.org/10.1182/blood-2009-05-222034 